spinulosa-associated polyomavirus in a fatal case of myocarditis in a seven-month-old girl. Int J Clin Exp Pathol. 2014;7:5308-12.

9. Siebrasse EA, Bauer I, Holtz LR, Le BM, Lassa-Claxton S, Canter C, et al. Human polyomaviruses in children undergoing transplantation, United States, 2008-2010. Emerg Infect Dis. 2012;18:1676-9. http://dx.doi.org/10.3201/eid1810.120359

10. Rockett RJ, Sloots TP, Bowes S, O'Neill N, Ye S, Robson J, et al. Detection of novel polyomaviruses, TSPyV, HPyV6, HPyV7, HPyV9 and MWPyV in feces, urine, blood, respiratory swabs and cerebrospinal fluid. PLoS One. 2013;8:e62764. http://dx.doi.org/ 10.1371/journal.pone.0062764

Address for correspondence: C. Patrick McClure, University of Nottingham, School of Life Sciences, Queen's Medical Centre, West Block A Floor, Nottingham, NG7 2UH, UK; email: patrick.mcclure@nottingham.ac.uk

\title{
Wohlfahrtiimonas chitiniclastica Bacteremia in Hospitalized Homeless Man with Squamous Cell Carcinoma
}

\section{Yuichi Katanami, Satoshi Kutsuna, Maki Nagashima, Saho Takaya, Kei Yamamoto, Nozomi Takeshita, Kayoko Hayakawa, Yasuyuki Kato, Shuzo Kanagawa, Norio Ohmagari}

Author affiliation: National Center for Global Health and Medicine, Tokyo, Japan

DOI: https://doi.org/10.3201/eid2409.170080

We report a case of Wohlfahrtiimonas chitiniclastica bacteremia in an elderly man in Japan who had squamous cell carcinoma. Blood cultures were initially negative for W. chitiniclastica but were positive on day 20. Careful attention needs to be paid to this organism in patients who have chronic wounds with maggots.

$\mathrm{W}_{\mathrm{i}}$ e report Wohlfahrtiimonas chitiniclastica bacteremia in a 75-year-old man in Japan who had squamous cell carcinoma on his shoulder. In September 2016, an unidentified patient was found unconscious on the ground by a passerby and admitted to the emergency department of the National Center for Global Health and Medicine (Tokyo, Japan). He had a necrotic lesion on his left shoulder with maggots. Blood analysis showed leukocytosis (26.61 $\times 10^{9}$ cells $/ \mathrm{L}$ [reference range $3.30-8.60 \times 10^{9}$ cells $\left./ \mathrm{L}\right]$ ), thrombocytosis $\left(626 \times 10^{9} / \mathrm{L}\right.$ [reference range 158-348 $\times$ $10^{9} / \mathrm{L}$ ]), anemia (hemoglobin, $9.6 \mathrm{~g} / \mathrm{dL}$ [reference range 13.7-6.8 $\mathrm{g} / \mathrm{dL}]$ ), and elevated C-reactive protein (87.9 $\mathrm{mg} / \mathrm{L}$ [reference range $0.00-1.40 \mathrm{mg} / \mathrm{L}$ ]). Albumin was $2.4 \mathrm{~g} / \mathrm{dL}$ (reference range $4.1-5.1 \mathrm{~g} / \mathrm{dL}$ ) and calcium was $12.6 \mathrm{mg} / \mathrm{dL}$ (reference range $8.8-10.1 \mathrm{mg} / \mathrm{dL}$ ). He was diagnosed with disturbance of consciousness caused by hypercalcemia and was hospitalized.

After saline infusion and intravenous cefazolin (3 $\mathrm{g} / \mathrm{d})$ were initiated, the patient's condition improved. A blood culture taken at the time of admission grew Peptoniphilus harei. A swab culture of the ulcer site grew Proteus mirabilis, Morganella morganii, and Kerstersia gyiorum. A biopsy was performed on day 3 , and the patient was diagnosed with squamous cell carcinoma. Enhanced computed tomography scanning revealed an ulcer and ring-enhancing lesion on his left shoulder (which was suspected of being a tumor or abscess) and multiple enlarged lymph nodes and $10-\mathrm{mm}$ pulmonary nodules in the right lung.

On day 20, the patient had fever and disturbance of consciousness; therefore, he was transferred to the Infectious Disease department of the hospital. Intravenous therapy with vancomycin $(1.5 \mathrm{~g} / \mathrm{d})$, cefepime $(3 \mathrm{~g} / \mathrm{d})$, and metronidazole $(1,500 \mathrm{mg} / \mathrm{d})$ was initiated, and the patient's fever and consciousness improved. Two cultures of blood taken on day 20 grew P. mirabilis, M. morganii, Streptococcus anginosus, Streptococcus agalactiae, Bacteroides fragilis, and gram-negative rods. After we obtained the culture results, vancomycin was stopped in accordance with the susceptibility test results. We identified the gramnegative rods as $W$. chitiniclastica by using matrix-assisted laser desorption/ionization time-of-flight mass spectrometry (Bruker Daltonics, Billerica, MA, USA), which showed scores of 2.239. We further confirmed the isolate to be $W$. chitiniclastica by using $16 \mathrm{~S}$ rRNA sequencing; the isolate was $99.08 \%$ identical to strain S5 (GenBank accession no. AM397063). We assessed the isolate's antimicrobial susceptibility profile (Table). The patient improved and was later discharged to another hospital.

W. chitiniclastica is a gram-negative, short, facultative anaerobic, straight-rod gammaproteobacterium that was first isolated from the parasitic fly Wohlfahrtia magnifica (1). This fly has not been reported in Japan. However, $W$. chitiniclastica has also been isolated from the Chrysomya megacephala fly, and this species has been reported in Japan (2), and from from the Musca domestica housefly, which is widely distributed all over the world (3). Campisi et al. reported that the Lucilia sericata fly might be a vector for $W$. chitiniclastica (4); this fly is common and widely distributed throughout Japan, and a 
Table. Antimicrobial susceptibility profile of Wohlfahrtiimonas chitiniclastica from a blood culture from a patient treated at the National Center for Global Health and Medicine, Tokyo, Japan, 2016.

\begin{tabular}{lc}
\hline Antimicrobial drug & MIC mg/L, susceptibility profile \\
\hline Piperacillin & $\leq 8, \mathrm{~S}$ \\
Piperacillin/tazobactam & $\leq 8, \mathrm{~S}$ \\
Ceftazidime & $\leq 4, \mathrm{~S}$ \\
Cefepime & $\leq 2, \mathrm{~S}$ \\
Aztreonam & $\leq 4, \mathrm{~S}$ \\
Imipenem/cilastatin & $\leq 1, \mathrm{~S}$ \\
Meropenem & $\leq 1, \mathrm{~S}$ \\
Amikacin & $\leq 8, \mathrm{~S}$ \\
Gentamicin & $\leq 2, \mathrm{~S}$ \\
Tobramycin & $\leq 2, \mathrm{~S}$ \\
Minocycline & $\leq 2, \mathrm{~S}$ \\
Levofloxacin & $1, \mathrm{~S}$ \\
Ciprofloxacin & $1, \mathrm{~S}$ \\
Trimethoprim/sulfamethoxazole & $\leq 2, \mathrm{~S}$ \\
${ }^{*}$ S, susceptible. &
\end{tabular}

case of cutaneous myiasis on skin cancer was reported (5). Unfortunately, we could not collect maggots from this patient because they were rapidly discarded at the emergency department.

Worldwide, few human cases of $W$. chitiniclastica infection have been documented. $W$. chitiniclastica has been described as a zoonotic pathogen ( $\sigma$ ) and reported from Hungary, Egypt, Niger, Germany, India, France, China, Argentina, Estonia, the United Kingdom, and the United States $(4,7,8)$. Rebaudet et al. (9) described the first human case of bacteremia attributable to $W$. chitiniclastica, which occurred in a 60-year-old homeless woman from southeastern France who had a history of alcoholism. Other human cases of $W$. chitiniclastica bacteremia were reported from Argentina (10) and the United Kingdom (4). Recently, a bacteremia case in a 72-year-old man was reported from Hawaii, USA (1).

Risk factors for $W$. chitiniclastica infection are poor personal hygiene, alcoholism, peripheral vascular disease, and chronic open wound (8). The patient we describe had a chronic wound because of squamous cell carcinoma, and the associated maggots were thought to be the transmission route. At admission, blood and swab cultures grew polymicrobial isolates without $W$. chitiniclastica, as confirmed by using matrix-assisted laser desorption/ ionization time-of-flight mass spectrometry. However, a blood culture on day 20 was positive for $W$. chitiniclastica. The patient probably was infected with $W$. chitiniclastica during hospitalization. Cases of $W$. chitiniclastica infection (with or without bacteremia) were reported as parts of polymicrobial infections $(1,4,7,8)$. W. chitiniclastica might have first infected this patient's ring-enhancing lesion as part of a polymicrobial infection. Because $W$. chitiniclastica was undetected in blood and swab cultures at admission, the organism might have entered the bloodstream during hospitalization.
This patient improved after intravenous therapy with cefepime and metronidazole. Previously reported $W$. chitiniclastica bacteremia cases were treated with combination antimicrobial therapies, including cefuroxime plus metronidazole plus clarithromycin (4), ceftazidime plus amikacin (10), piperacillin-tazobactam plus clindamycin plus vancomycin (1), ceftriaxone monotherapy (9), and meropenem monotherapy (1). Two of the 5 cases were fatal $(1,10)$.

Clinicians should be attentive to the possibility of $W$. chitiniclastica infection in patients who have chronic wounds with maggots and poor hygiene. Clinical suspicion is warranted even if blood and swab cultures are initially negative for $W$. chitiniclastica.

This work was supported by a grant from Japan's National Center for Global Health and Medicine (grant no. 29-1018).

\section{About the Author}

Dr. Katanami is a medical doctor at the National Center for Global Health and Medicine, Disease Control Prevention Center. His main research interest is tropical infectious diseases.

\section{References}

1. Nogi M, Bankowski MJ, Pien FD. Wohlfahrtiimonas chitiniclastica infections in 2 elderly patients, Hawaii, USA. Emerg Infect Dis. 2016;22:567-8. http://dx.doi.org/10.3201/eid2203.151701

2. Mihara M, Kurahashi H. Base-line susceptibility of the oriental latrine fly, Chrysomya megacephala (Diptera: Calliphoridae), to five insecticides. Med Vet Entomol. 1991;5:51-4. http://dx.doi.org/10.1111/j.1365-2915.1991.tb00520.x

3. Gupta AK, Nayduch D, Verma P, Shah B, Ghate HV, Patole MS, et al. Phylogenetic characterization of bacteria in the gut of house flies (Musca domestica L.). FEMS Microbiol Ecol. 2012;79:58193. http://dx.doi.org/10.1111/j.1574-6941.2011.01248.x

4. Campisi L, Mahobia N, Clayton JJ. Wohlfahrtiimonas chitiniclastica bacteremia associated with myiasis, United Kingdom. Emerg Infect Dis. 2015;21:1068-9. http://dx.doi.org/ 10.3201/eid2106.140007

5. Chigusa Y, Shinonaga S, Kawaguchi T, Kawaguchi N, Kirinoki M, Matsuda H. Cutaneous myiasis caused by Lucilia sericata (Diptera: Calliphoridae) on skin cancer of the cheek. Medical Entomology and Zoology. 2002;53:89-94. http://dx.doi.org/ 10.7601/mez.53.89 1

6. Thaiwong T, Kettler NM, Lim A, Dirkse H, Kiupel M. First report of emerging zoonotic pathogen Wohlfahrtiimonas chitiniclastica in the United States. J Clin Microbiol. 2014;52:2245-7. http://dx.doi.org/10.1128/JCM.00382-14

7. Kõljalg S, Telling K, Huik K, Murruste M, Saarevet V, Pauskar M, et al. First report of Wohlfahrtiimonas chitiniclastica from soft tissue and bone infection at an unusually high northern latitude. Folia Microbiol (Praha). 2015;60:155-8. http://dx.doi.org/10.1007/ s12223-014-0355-x

8. de Dios A, Jacob S, Tayal A, Fisher MA, Dingle TC, Hamula CL. First report of Wohlfahrtiimonas chitiniclastica isolation from a patient with cellulitis in the United States. J Clin Microbiol. 2015;53:3942-4. http://dx.doi.org/10.1128/JCM.01534-15

9. Rebaudet S, Genot S, Renvoise A, Fournier PE, Stein A. Wohlfahrtiimonas chitiniclastica bacteremia in homeless woman. 
Emerg Infect Dis. 2009;15:985-7. http://dx.doi.org/10.3201/ eid1506.080232

10. Almuzara MN, Palombarani S, Tuduri A, Figueroa S, Gianecini A, Sabater L, et al. First case of fulminant sepsis due to

Wohlfahrtiimonas chitiniclastica. J Clin Microbiol. 2011;49:23335. http://dx.doi.org/10.1128/JCM.00001-11

Address for correspondence: Yuichi Katanami, National Center for Global Health and Medicine, Disease Control and Prevention Center, 1-21-1, Toyama, Shinjuku, Tokyo 162-8655, Japan; email: yuichi.katanami@gmail.com

\section{Symptomatic Dengue during Pregnancy and Congenital Neurologic Malformations}

\author{
Enny S. Paixão, Maria Glória Teixeira, \\ Maria da Conceição N. Costa, \\ Mauricio L. Barreto, Laura C. Rodrigues
}

\begin{abstract}
Author affiliations: London School of Hygiene and Tropical Medicine, London, United Kingdom (E.S. Paixão, L.C. Rodrigues); Instituto de Saúde Coletiva, Salvador-Bahia, Brazil (E.S. Paixão, M.G. Teixeira, M. da Conceição N. Costa, M.L. Barreto); Center of Data and Knowledge Integration for Health (CIDACS), Salvador-Bahia (M.L. Barreto, L.C. Rodrigues)
\end{abstract}

DOI: https://doi.org/10.3201/eid2409.170361

Dengue virus infection during pregnancy increased the risk for any neurologic congenital anomaly in the infant by roughly $50 \%$ and for other congenital malformations of brain 4-fold. Our results show an association between dengue during pregnancy and congenital anomalies of the brain, suggesting that flaviviruses other than Zika virus are associated with such malformations.

$\mathrm{B}$ efore the causal relationship between Zika virus and neurologic congenital anomalies (1), especially microcephaly (2), was established, no evidence associated flavivirus with congenital malformations in humans, although postnatal complications have been described (3). We investigated whether dengue virus (DENV) infection during pregnancy could be associated with neurologic defects in the infant at birth.

We conducted a population-based study using routinely collected data from live births and from women who were notified and confirmed to have DENV infection during 2006-2012 in Brazil, before the introduction of Zika virus. We probabilistically linked records of mothers of live births with records of dengue notification to identify women who were reported as having dengue during pregnancy. We excluded records with missing or implausible names, multiple pregnancies, and births in municipalities with no dengue notifications. We obtained ethics approval from Federal University of Bahia, Salvador, Brazil (CAAE: 26797814.7.0000.5030) and from London School of Hygiene and Tropical Medicine (Ethics Ref:10269).

In the matching process, we used name, age, and place of residence of the mother at time of delivery and notification. We included only links and nonlinks with a high degree of certainty. We validated the linkage process in a study that demonstrated $62 \%$ sensitivity (4).

We used an outcome definition of congenital malformation of the nervous system coded as Q00-Q07 in International Classification of Diseases, 10th Revision (ICD-10). We defined dengue as a confirmed case of DENV infection notified during a pregnancy that resulted in a live birth. We estimated the association between symptomatic dengue during pregnancy and neurologic congenital malformations using the Firth method to reduce the small sample bias in maximum-likelihood estimation.

The study parameters encompassed 16,103,312 live births. Neurologic congenital anomalies are rare; they occurred in 13,634 (0.08\%) live births. Dengue during pregnancy increased the odds of a neurologic congenital anomaly by $50 \%$ (Table), but this result was not statistically significant (95\% CI $0.97-2.27)$. We split the neurologic congenital defects into ICD-10 categories; the 95\% $\mathrm{CI}$ around the estimated odds ratios (ORs) was not statistically significant in 7 categories, including microcephaly (OR 1.7, 95\% CI 0.33-8.32). Two other types of neurologic congenital anomalies were $>4$ times more frequent in women who had DENV infection during pregnancy: other congenital malformations of spinal cord (OR 5.4, 95\% CI 1.0-26.9) and other congenital malformations of brain (OR 4.5, 95\% CI 1.7-11.3, which was statistically significant). We found no sign of space-time clusters or recording errors suggestive of a coding artifact in the 4 records of other congenital malformations of brain wherein the mother had DENV infection (online Technical Appendix Table, https://wwwnc.cdc.gov/EID/article/24/9/17-0361Techapp1.pdf)

Symptoms of DENV infection occurred in the first trimester in $50 \%$ of patients. The specific diagnosis of those among the nonexposed group were congenital malformation of corpus callosum $(9 \% ; 81 / 943)$, holoprosencephaly $(24 \% ; 225 / 943)$, and septooptic dysplasia $(0.6 \% ; 6 / 943)$.

Our study showed an association between DENV infection during pregnancy and congenital anomalies of the 